\title{
EDITORIAL
}

\section{Noncommunicable Disease (NCDs) Burden of Bangladesh}

(Birdem Med J 2012; 2(1): 3)

A recent study by Ministry of health and family welfare Govt of Bangladesh shows that the burden of NCDs now surpasses infectious diseases in the country and now account for $61 \%$ of all adult deaths in Bangladesh. The Bangladesh NCD risk factor survey 2010 showed 60 million people in the country consume less than the recommended amonut of fruit and vegetables, 16 million leads sedentary lives and 33 million people use tobacco.

Conventional NCDS are-CVDs(cardiovascular and cerebrovascular diseases), Diabetes mellitus, COPD, Cancer, Renal diseases, Dafness, Arsenicosis, osteoporosis, Oral health and Thalassemias.while RTA (road traffic accident,Occupational hazards,Postdisaster health, Health hazards due to change of climate,Mental health and hazards use of Tobacco and substance abuses are componenets of nonconventional NCDS. 2010 National NCD risk factor survey showed $99 \%$ of those surveyed had at least one NCD risk factor and $29 \%$ had more than 3 risk factors
Major NCDS of Bangladesh include Diabetes Mellitus (DM), Iscemic heart disease (IHD), hypertension (HTN), Stroke, Chronic respiratory disease and cancer.

IHD tops the NCDs in our country, while DM is rising in alarming rate. Prevalence of DM in urban and rural populations are $10 \%$ and $7 \%$ respectively.Prevalence of Hypertension is $9.8 \%$ in males and $15.6 \%$ among females. Cancer claimed 150000 lives according to.cancer institute estimate in 2007. Five major primary sites of cancer include Respiratry system and thorax( $22.2 \%$ ), Digestive system (20.8\%), Breast (12.7\%), Female genital organ (12.1\%), lip, Oral cavity and Pharynx (10.9\%).

Last time Goverment health sector programmes were designed mostly on the basis of MDGs where NCDs were not prioritized. Next health sector programme (2011-2016) includes a separate operational (OP) plan to prevent, mange and control of NCDs 